\title{
In Vivo Cytochrome P450 3A Isoenzyme Activity and Pharmacokinetics of Imatinib in Relation to Therapeutic Outcome in Patients With Chronic Myeloid Leukemia
}

\author{
Karin Skoglund, Johan Richter, Ulla Olsson-Stromberg, Jonas Bergquist, Warunika \\ Aluthgedara, S. J. Kumari A. Ubhayasekera, Svante Vikingsson, Anna Svedberg, Stina \\ Soderlund, Anna Sandstedt, Anders Johnsson, Jesper Aagesen, Jonas Alsenhed, Staffan \\ Hägg, Curt Peterson, Kourosh Lotfi and Henrik Green
}

\section{Linköping University Post Print}

\section{Tweet}

N.B.: When citing this work, cite the original article.

Original Publication:

Karin Skoglund, Johan Richter, Ulla Olsson-Stromberg, Jonas Bergquist, Warunika Aluthgedara, S. J. Kumari A. Ubhayasekera, Svante Vikingsson, Anna Svedberg, Stina Soderlund, Anna Sandstedt, Anders Johnsson, Jesper Aagesen, Jonas Alsenhed, Staffan Hägg, Curt Peterson, Kourosh Lotfi and Henrik Green, In Vivo Cytochrome P450 3A Isoenzyme Activity and Pharmacokinetics of Imatinib in Relation to Therapeutic Outcome in Patients With Chronic Myeloid Leukemia, 2016, Therapeutic Drug Monitoring, (38), 2, 230-238. http://dx.doi.org/10.1097/FTD.0000000000000268

Copyright: Lippincott, Williams \& Wilkins

\section{http://www.lww.com/}

Postprint available at: Linköping University Electronic Press

http://urn.kb.se/resolve?urn=urn:nbn:se:liu:diva-129678 

Title: In vivo CYP3A activity and pharmacokinetics of imatinib in relation to therapeutic outcome in chronic myeloid leukemia patients

Authors: Karin Skoglund, $\mathrm{PhD}^{1}$, Johan Richter, Dr. Med, $\mathrm{PhD}^{2}$, Ulla Olsson-Strömberg, Dr.Med, PhD ${ }^{3}$, Jonas Bergquist $\mathrm{PhD}^{4}$, Warunika Aluthgedara, $\mathrm{MSc}^{4}$, S. J. Kumari A. Ubhayasekera, $\mathrm{PhD}^{4}$, Svante Vikingsson, $\mathrm{PhD}^{1,5}$, Anna Svedberg, MSc. ${ }^{1}$, Stina Söderlund, Dr. Med ${ }^{3}$, Anna Sandstedt Dr. Med ${ }^{6}$, Anders Johnsson Dr. Med $^{7}$, Jesper Aagesen Dr. Med ${ }^{8}$, Jonas Alsenhed Dr. Med ${ }^{9}$, Staffan Hägg, Dr. Med, PhD¹, Curt Peterson, Dr. Med, PhD ${ }^{1}$, *Kourosh Lotfi, Dr. Med, PhD ${ }^{1,6}$, *Henrik Gréen PhD ${ }^{1,10}$

${ }^{*} \mathrm{HG}$ and KL share the last authorship

\section{Affiliations:}

${ }^{1}$ Division of Drug Research/Clinical Pharmacology, Department of Medical and Health Sciences, Linköping University, Linköping, Sweden

2Department of Hematology and Coagulation, Skåne University Hospital, Lund, Sweden

${ }^{3}$ Department of Medical Sciences, Uppsala University and Department of Hematology, University Hospital, Uppsala, Sweden

${ }^{4}$ Analytical Chemistry, Department of Chemistry - Biomedical Center and Science for Life Laboratory, Uppsala University, Uppsala, Sweden

${ }^{5}$ Science for Life Laboratory, Division of Translational Medicine and Chemical Biology, Department of Medical Biochemistry and Biophysics, Karolinska Institutet, Stockholm, Sweden

${ }^{6}$ Department of Hematology, Linköping University Hospital, Linköping, Sweden

${ }^{7}$ Department of Internal Medicine, Motala Hospital, Motala, Sweden

${ }^{8}$ Department of Medicine, Ryhov County Hospital, Jönköping, Sweden

${ }^{9}$ Department of Internal Medicine, Västervik Hospital, Västervik, Sweden

${ }^{10}$ Department of Forensic Genetics and Forensic Toxicology, National Board of Forensic Medicine, Linköping, Sweden 


\section{Corresponding author and request for reprints:}

Henrik Gréen

Address: Division of Drug Research/Clinical Pharmacology, Department of Medical and Health Sciences, Linköping University, 58185 Linköping, Sweden

Email: henrik.green@liu.se, Phone: +46 732701227, Fax: +46 13136005

\section{Conflicts of interest and source of funding:}

This study was funded by the Swedish Research Council, the Swedish Cancer Society and the Medical Research Council of Southeast Sweden. KL and HG received an academic grant from Novartis for funding of the study. Novartis also provided imatinib mesylate and CGP74588. JR has received honoraria and research support from Novartis, but not in relation to this project. The remaining authors have no conflict of interests or funding to declare. 


\begin{abstract}
Background: CYP3A metabolic activity varies between individuals and is therefore a possible candidate of influence on the therapeutic outcome of the tyrosine kinase inhibitor imatinib in chronic myeloid leukemia (CML) patients. The aim of this study was to investigate the influence of CYP3A metabolic activity on the plasma concentration and outcome of imatinib in CML patients.
\end{abstract}

Methods: Forty-three CML patients were phenotyped for CYP3A activity using quinine as a probe drug and evaluated for clinical response parameters. Plasma concentrations of imatinib and its main metabolite, CGP74588, were determined using liquid chromatography-mass spectrometry.

Results: Patients with optimal response to imatinib after 12 months of therapy did not differ in CYP3A activity compared to non-optimal responders (quinine metabolic ratio of 14.69 and 14.70, respectively; $\mathrm{P}=0.966$ ). Neither the imatinib plasma concentration nor the CGP74588/imatinib ratio was significantly associated with CYP3A activity.

Conclusions: CYP3A activity does not influence imatinib plasma concentrations or the therapeutic outcome. These results indicate that even though imatinib is metabolized by CYP3A enzymes, this activity is not the ratelimiting step in imatinib metabolism and excretion. Future studies should focus on other pharmacokinetic processes so as to identify the major contributor to patient variability in imatinib plasma concentrations.

Keywords: pharmacokinetics, chronic myeloid leukemia, imatinib, CGP74588, CYP3A 


\section{Introduction}

The tyrosine kinase inhibitor (TKI) imatinib is approved for first-line treatment of chronic myeloid leukemia (CML) in chronic and accelerated phases. Even though the majority of CML patients experience an adequate therapeutic effect, 26-37\% of patients discontinue imatinib therapy due to a suboptimal response or intolerance. ${ }^{1,2}$ With the approval of the second generation TKIs (dasatinib and nilotinib) for first-line treatment of CML, additional therapeutic options have become available. In order to improve CML treatment further, a more personalized treatment strategy might be appropriate in which imatinib is administered only to patients for whom an optimal response to this TKI can be predicted. To achieve this aim, a better understanding of the mechanisms of response and resistance to imatinib is warranted.

A number of factors can influence the effect of imatinib therapy, including pharmacokinetic determinants that influence the exposure of target cells to the drug. Imatinib is extensively metabolized by the hepatic cytochrome P450 3A (CYP3A) isoenzyme subfamily., ${ }^{3,4}$ In addition, imatinib is a substrate for the ATP-binding cassette transporters ABCB1 and ABCG2 ${ }^{5,6}$ and the organic cation transporter 1 (OCT-1) ${ }^{7}$ that are expressed in the liver. ${ }^{8-10}$ These transporters can potentially influence the uptake, efflux, and availability of imatinib for metabolism and excretion.

Imatinib plasma trough concentrations above $1000 \mathrm{ng} / \mathrm{mL}$ have been associated with enhanced response rates, ${ }^{11}$,

12 but the inter-individual variation in imatinib concentration at steady-state at a standard $400 \mathrm{mg} /$ day dose regimen is substantial and a 25 -fold difference between high and low values has been observed. ${ }^{11}$ The observed variation in plasma concentrations might be due to the fact that CYP3A enzymes have a considerable interindividual variation in metabolic activity. ${ }^{13,14}$

We have previously reported the results from a pilot study on 14 CML patients in which it was found that CYP3A metabolic activity was inversely associated with the therapeutic effects of imatinib indicating the importance of metabolites in imatinib treatment. ${ }^{15}$ The present study was designed to follow up the previous finding and had the primary aim of investigating the influence of in vivo CYP3A activity on the outcome of first-line imatinib therapy in chronic-phase CML patients. The secondary aim was to study the influence of CYP3A activity on hematologic toxicity during imatinib treatment and the trough plasma concentrations of imatinib and its pharmacologically active metabolite, CGP74588.

\section{Materials and methods}




\section{Patients and study design}

The mean CYP3A activity in responding vs. non-responding groups from the pilot study was used for power calculations preceding the present study. To reproduce the difference in means between the groups in the pilot study with a power of $80 \%$, a minimum of 7 patients in each group was predicted. To achieve a sufficient number of non-responders to imatinib treatment, we aimed to include 50 patients in total. Patients with chronicphase CML at diagnosis, and receiving first-line treatment with $400 \mathrm{mg} /$ day imatinib, were eligible for the study. Imatinib dose adjustments, as well as pre-treatment with interferon, hydroxyurea, or allopurinol were allowed for inclusion. Exclusion criteria were significant treatment before or during the first year of imatinib therapy such as transplantation or the use of other drugs than those mentioned above, on-going treatments, or concurrent diseases that were contraindicated with quinine. Study subjects could be enrolled either retrospectively (after the start of imatinib therapy) or prospectively (before the initiation of imatinib). Information regarding response parameters and adverse reactions were collected from patient medical records.

This study was approved by the Regional Ethical Review Board in Linköping, Sweden (No: 02-221) and all patients gave their informed consent prior to their inclusion in the study.

\section{Response parameters}

The primary endpoint of the study was the cytogenetic and molecular responses 12 months after the start of imatinib treatment. Cytogenetic and molecular responses were evaluated from routine samplings documented in patient medical records. Cytogenetic response was determined by the fraction of Philadelphia chromosome positive $\left(\mathrm{Ph}^{+}\right)$metaphases out of 7-35 (mean $\left.=22.7\right)$ analyzed metaphases in bone marrow aspirates. A complete cytogenetic response (CCgR) was defined as the absence of $\mathrm{Ph}^{+}$metaphases. Molecular response (MR) was evaluated using real-time PCR in which the percentage of $B C R-A B L$ gene transcripts compared to a housekeeping gene was determined. Samples in the present study were analyzed in three different laboratory facilities during the years 2004-2012. Due to the time span of samplings, only 13 patients (35\%) out of the 37 with an evaluable MR had been evaluated according to the standardized International Scale. ${ }^{16}$ According to the European LeukemiaNet guidelines, CCgR is defined as optimal response after 12 months of therapy. Other investigators have found that the achievement of an MR of $B C R-A B L<1 \%$ gives the same predictive power as CCgR. ${ }^{17,} 18$ Because not all patients had documented information on cytogenetic response at 12 months, we defined optimal response here as the achievement of CCgR or $B C R-A B L<1 \%$ within 12 months \pm 30 days. Patients who stopped imatinib therapy before the end of the first year due to suboptimal response were classified 
as non-optimal responders. In addition to the optimal response defined here, the traditional CCgR and major molecular response (MMR) of $B C R-A B L<0.1 \%$ were evaluated separately.

\section{Hematologic toxicity}

The results of routine blood tests recorded in patient medical records during the first year of imatinib treatment were examined for the identification and classification of hematologic toxicities. Total white blood cells, hemoglobin, neutrophil, and platelet levels were evaluated and graded according to the National Cancer Institute Common Terminology Criteria for Adverse Events version 3.0 in which events are classified as grade 0 to 5 with grade 5 being the most serious event (death).

Drugs and chemicals

Quinine, formulated as 250 mg capsules, was administered to study subjects (Recip AB, Solna, Sweden). For analytical purposes, standard samples of quinine and 3S-3-hydroxy quinine (3S-Q) were obtained from Toronto Research Chemicals, North York, Canada, and 3R-3-hydroxy quinine was from Santa Cruz Biotechnology, Heidelberg, Germany. Imatinib mesylate and CGP74588 were kindly provided for analytical purposes by Novartis Pharma AG (Basel, Switzerland). Trazodone was obtained from Sigma Aldrich (Stockholm, Sweden).

Phenotyping of CYPЗA enzyme activity in vivo

Quinine has previously been validated as a probe drug that can be used to estimate CYP3A activity in vivo by the quantification of quinine and its CYP3A metabolite 3S-Q in plasma. ${ }^{19}$ Quinine is metabolized by CYP3A4 and CYP3A5, ${ }^{20,21}$ which constitute the CYP3A metabolic activity in adults. ${ }^{22}$ All patients were phenotyped once, and the prospectively included patients were phenotyped both before and three months after the start of imatinib treatment.

Patients were given $250 \mathrm{mg}$ of quinine per oral administration and whole blood was drawn $16 \mathrm{~h} \pm 2 \mathrm{~h}$ after intake. The blood sample was centrifuged at $3000 \mathrm{~g}$ for $10 \mathrm{~min}$ and plasma was extracted and stored at $-20{ }^{\circ} \mathrm{C}$ or $-70{ }^{\circ} \mathrm{C}$ until analysis. The conversion of quinine into 3S-Q by CYP3A was assessed by the quantification of quinine and 3S-Q in patient plasma using an HPLC assay adapted from a previously published method ${ }^{23}$ with the following changes. Samples were analyzed on an HPLC system consisting of an Alliance 2695 Separations module (Waters) connected to a Multi lambda Fluorescence Detector 2475 using an xBridge C18 column (150 × $3 \mathrm{~mm}, 3.5 \mu \mathrm{m}$ (Waters)) kept at a constant temperature of $60^{\circ} \mathrm{C}$. Patient plasma was precipitated and $10 \mu \mathrm{L}$ were 
injected onto the column. The two mobile phases A (10\% acetonitrile in acetate buffer (88 mM acetic acid and $12 \mathrm{mM}$ ammonium acetate)) and B (26\% acetonitrile in acetate buffer) were delivered at $0.8 \mathrm{~mL} / \mathrm{min}$ and mixed according to the following gradient profile: 0-5 min, a linear increase from 0\% B to 25\% B; 5-9.4 min, a linear increase from 25\% B to $100 \% \mathrm{~B}$; and 9.4-12.5 min, re-equilibration to 0\% B.

The chromatographic separation of 3S-Q from its diastereomer 3R-3-hydroxyquinine was confirmed. Calibration points were spiked in plasma in the concentration ranges of $100 \mathrm{nM}$ to $20000 \mathrm{nM}$ for quinine and $10 \mathrm{nM}$ to $2000 \mathrm{nM}$ for 3S-Q. Quality control samples were prepared at three concentrations (300 nM, $3000 \mathrm{nM}$, and $10000 \mathrm{nM}$ for quinine and $30 \mathrm{nM}, 300 \mathrm{nM}$, and $1000 \mathrm{nM}$ for 3S-Q) and analyzed in five replicates to determine the reliability of the assay. Assay coefficients of variation within and between days for all investigated quality control concentrations were $<3.9 \%$ for both quinine and 3S-Q and the accuracy ranged from $99.5 \%$ to $109 \%$.

Patient samples were analyzed in duplicate and the concentrations of quinine and 3S-Q were calculated from the respective standard curves. The ratio of quinine and 3S-Q concentrations was calculated and the mean ratios of the duplicate measurements were used as the measure of CYP3A activity.

\section{Quantification of imatinib and CGP74588 in plasma}

Patients were analyzed for steady state trough levels of imatinib and CGP74588 in plasma both at the time of CYP3A phenotyping and on one additional occasion when quinine was not co-administered. In patients included prospectively, plasma concentrations were evaluated at one, three, and six months after start of imatinib therapy in order to evaluate the effects of a potential change in CYP3A metabolic activity over time.

Imatinib was given once daily and whole blood from patients on imatinib treatment was drawn $24 \mathrm{~h} \pm 2 \mathrm{~h}$ after the last drug intake and before the intake of next dose. Blood was centrifuged at $1350 \mathrm{~g}$ for 10 min followed by plasma extraction and storage at $-20^{\circ} \mathrm{C}$ or $-70^{\circ} \mathrm{C}$ until analysis. Each plasma sample $(100 \mu \mathrm{L})$ was mixed with $10 \mu \mathrm{L}$ of the internal standard (IS) trazodone in methanol $(10 \mu \mathrm{g} / \mathrm{mL})$. Methanol $(190 \mu \mathrm{L})$ was added to each tube and vortexed for $20 \mathrm{~min}$. The sample was centrifuged at $4{ }^{\circ} \mathrm{C}$ for $10 \mathrm{~min}$ at $14000 \mathrm{~g}$. The resulting supernatant was transferred to autosampler vials and $5 \mu \mathrm{L}$ were injected into a liquid chromatography-mass spectrometry system.

The liquid chromatography system consisted of an Agilent model 1100 Auto-sampler (Agilent Technologies, Palo Alto, CA, USA), an Agilent 1100 Quaternary pump, and a Kinetex XB C18 column (5 $\mu \mathrm{m}, 2.1 \mathrm{~mm} \times 50$ 
mm (Phenomenex, Torrance, CA, USA) controlled by the Agilent ChemStation software (Agilent Technologies). Imatinib and CGP74588 in patient plasma were separated using a gradient elution profile adapted from a previously published method ${ }^{24}$. The mobile phase run at a linear gradient containing $0.1 \%$ formic acid in both methanol and water with the flow rate starting from $0.2 \mathrm{~mL} / \mathrm{min}$. The total run time was 14 min. The chromatographic system was coupled to a time of flight (TOF) mass spectrometer (Agilent Technologies) equipped with electrospray ionization interphase (ESI). The detection was performed in positive ion mode. The ionization potential was $3800 \mathrm{~V}$ and the ion source temperature was $300{ }^{\circ} \mathrm{C}$. The nebulizing gas was used for ESI at a pressure of 15 psi. The voltages fixed at the fragmenter, skimmer, and octopole guides were $225 \mathrm{~V}, 60 \mathrm{~V}$, and $250 \mathrm{~V}$, respectively. The ion pulser at the TOF analyzer was set at a measurement frequency of two cycles/s. Peak lists were obtained with the molecular feature extractor software "MassHunter" (Agilent Technologies). Extraction of total ion chromatograms for imatinib, CGP74588, and trazodone were done with $\mathrm{m} / \mathrm{z}$ ranges of 494.15-494.3, 480.2-480.3, and 372.1-372.2, respectively. Typical chromatograms and mass spectra of the analytes in patient plasma are shown in Supplemental Digital Content 1 (Figure S1).

The calibration curves were prepared individually by plotting the peak area of imatinib and CGP74588, normalized to IS peak area, versus the nominal concentrations of imatinib and CGP74588. Calibration curves were prepared in the ranges of 30-7000 ng/mL for imatinib and 30-3000 ng/mL for CGP74588. The linearity was determined by linear regression analysis and the correlation coefficients $\left(\mathrm{r}^{2}\right)$ of imatinib and CGP74588 were 0.9944 and, 0.9994 respectively. Quality control samples were prepared at three concentrations for intraand inter-assay validation of the method $(100,1000$, and $2000 \mathrm{ng} / \mathrm{mL}$ for imatinib and 30, 100, and $500 \mathrm{ng} / \mathrm{mL}$ for CGP74588). Assay coefficients of variation were found to be $<8 \%$ with accuracies ranging $93 \%-110 \%$ for both compounds. Patient samples were analyzed in duplicate. Imatinib and CGP74588 peak areas were normalized to the IS peak area and quantities were calculated from the calibration curve. Plasma concentrations determined as ng/mL were converted into $\mu \mathrm{M}$ for calculation of CGP74588/imatinib metabolic ratios.

\section{Genotyping}

Genomic DNA from patients was extracted from whole blood using the Maxwell 16 Blood DNA Purification Kit (Promega, Madison, WI, USA) on the Maxwell 16 instrument (Promega) according to the manufacturer’s instructions. Two hundred fifty nanograms of DNA were used in the multiplex genotyping assay iPLEX ADME PGx panel and analyzed with the MALDI-TOF-based MassARRAY system (Sequenom, San Diego, CA, USA). The Sequenom ADME panel includes assays for numerous genetic variations in genes potentially influencing 
drug absorption, distribution, metabolism, and excretion although only genetic variations in ABCG2 and OCT-1 were analyzed for the present study. The complete details of analyzed variants are summarized in Supplemental Digital Content 2, Table S1.

\section{Statistical analysis}

Differences in CYP3A activity or plasma drug concentrations within the same individuals at different time points were analyzed with the paired sample $t$-tests. Differences in CYP3A activity or plasma concentrations in groups based on response, hematologic toxicity, or genotype were analyzed with the non-parametric MannWhitney $U$-test for less than three groups or the Kruskal-Wallis test for more than two analyzed groups. The influence of gender on CYP3A activity was analyzed using an independent sample, 2-tailed $t$-test. All analyses were performed using IBM SPSS Statistics 19 (IBM, Armonk, NY, USA). The correlations between scaled parameters such as CYP3A activity, age, and plasma drug concentration were analyzed using a linear regression model in GraphPad Prism 5 (GraphPad Software, La Jolla, CA, USA). $P$-values $<0.05$ were considered significant.

\section{Results}

Patient characteristics and response to imatinib therapy

A total of 55 patients were included in the study, of which 20 were women. The median age was 60 years (range: 18-87 years) and the median age at the time of CML diagnosis was 55 years (range: 10-83 years). Sokal scores were evaluable for 43 patients of whom 23\%, 37\%, and 40\% were low-, intermediate-, and high-risk patients, respectively.

Twelve patients were excluded from the analysis of response to imatinib due to following reasons: patients received significant treatment other than imatinib either before or in combination with imatinib $(n=4)$; the patient had reached accelerated phase prior to the start of imatinib $(n=1)$; the patient had no evaluable response parameter $(n=1)$; patients stopped imatinib before the end of 12 months due to adverse events $(n=2)$; or patients were evaluated for imatinib outcome in the pilot study $(n=4)$. Thus, the remaining 43 patients were evaluable for outcome of imatinib therapy. Patients were commonly pre- or co-treated with allopurinol or hydroxyurea, and one patient had been treated with interferon for eight months prior to imatinib. An optimal response of CCgR or $B C R-A B L<1 \%$ was achieved in $77 \%(n=33)$ of the patients within 12 months of imatinib therapy. One patient was considered an optimal responder due to the achievement of $B C R-A B L<1 \%$ but did not 
achieve CCgR. This was the only discrepancy between CCgR and $B C R-A B L<1 \%$ in our material. Ten patients did not achieve an optimal response, of whom seven stopped imatinib therapy during the first 12 months due to suboptimal response and three were still on imatinib therapy at the end of 12 months but did not reach CCgR or $B C R-A B L<1 \%$. In six out of the ten non-responding patients, information on $B C R-A B L 1$ mutation status was available but none carried the T315I mutation. Studying the cytogenetic and MR parameters separately, 38 and 37 patients had evaluable cytogenetic response and MR at the 12-month time point, respectively. Out of these patients, 71\% reached a CCgR and 49\% had an MMR.

CYP3A activity and influence on therapeutic outcome

The CYP3A activity had a 5-fold variation with quinine/3S-Q metabolic ratios ranging from 6.8 to 34 in the total study population of 55 patients. Neither age nor gender had any significant influence on the observed CYP3A activity (data not shown).

The CYP3A phenotyping was performed at any time after the start of imatinib therapy. The median time from start of imatinib to CYP3A phenotyping in the 43 patients evaluated for imatinib outcome was 31 months (range: 1 day-119 months). Optimally responding patients had a very similar median CYP3A activity of 14.69 compared to 14.70 in the group of patients who did not achieve this response level and no significant difference in CYP3A activity between the groups was detected (Fig 1a). Analyzing the separate response parameters of MMR and CCgR gave similar results (Fig 1b-c). Patient medical records were examined for co-treatment with drugs potentially influencing CYP3A activity at the time of CYP3A phenotyping. None of the previously identified inhibitors or inducers ${ }^{25,}{ }^{26}$ were found even though other CYP3A substrates were co-administered in a number of cases.

\section{CYP3A activity and hematologic toxicity}

The analysis of CYP3A influence on hematologic toxicity was restricted to patients who were treated with 400 $\mathrm{mg}$ /day imatinib at the time of toxicity or for the rest of the first year if no toxicity occurred. In 36 patients, these criteria were fulfilled and eight patients had grade 3-4 hematologic toxicity. No patient died from toxicity (grade 5). No difference in CYP3A activity was detected between the groups that did and did not experience grade 3-4 toxicity (Fig 2).

CYP3A activity and plasma concentrations 
In order to investigate the potential influence of co-administering quinine for CYP3A phenotyping on imatinib and CGP74588 plasma concentrations, samples taken with and without the presence of quinine were compared. Out of the 55 patients included in the study, 33 had evaluable imatinib samplings with and without the coadministration of quinine. Patients had a mean imatinib concentration of $1052 \mathrm{ng} / \mathrm{mL}$ with concomitant quinine administration, and this was significantly lower compared to $1281 \mathrm{ng} / \mathrm{mL}$ without quinine $(P=0.032)$. A significant influence was also detected on the CGP74588/imatinib ratio, which was higher in the presence of quinine $(P<0.000)$. The CGP74588 concentration with quinine was higher than without quinine, but the difference did not reach statistical significance. Because of the possible interaction between quinine and imatinib metabolism, samplings without co-administration of quinine were used for further evaluation of imatinib and CGP74588 plasma concentrations.

Out of the 55 patients in the total study population, imatinib and CGP74588 trough concentrations were evaluable in 34 patients without the co-administration of quinine. The 21 patients omitted from the analysis were excluded because blood sampling took place outside the $24 \mathrm{~h} \pm 2 \mathrm{~h}$ interval after intake of the drug ( $\mathrm{n}=$ 10); the patient was no longer on imatinib treatment at the time of CYP3A phenotyping $(n=8)$; or the patient did not have samplings without the co-administration of quinine $(n=3)$. Three of the 34 patients analyzed for plasma concentrations had received doses of imatinib other than $400 \mathrm{mg} / \mathrm{day}$ (100 mg/day, n = 1; $800 \mathrm{mg} / \mathrm{day}, \mathrm{n}$ $=2$ ), and imatinib plasma concentrations were dose normalized in these cases. The mean imatinib concentration was 1273 ng/mL (range: 509-3485 ng/mL). CGP74588 quantities could not be accurately dose normalized and the mean CGP74588 concentration in the 31 patients treated with $400 \mathrm{mg} /$ day imatinib was $322 \mathrm{ng} / \mathrm{mL}$ (range: 65-829 ng/mL). The ratio of CGP74588 to imatinib was determined in the complete set of the 34 evaluable patients and the mean ratio was 0.23 (range: $0.13-0.68$ ).

The correlation between CYP3A phenotype, imatinib, and the CGP74588/imatinib ratio in plasma was investigated in the set of 34 patients. No significant association between CYP3A metabolic activity and imatinib concentrations was identified (Fig 3a) nor was there any correlation between CYP3A activity and the CGP74588/imatinib ratio (Fig 3b).

\section{Results from prospectively studied patients}

Eight patients were prospectively studied, of which seven were CYP3A phenotyped before the start of imatinib therapy and at three months afterwards. In terms of response to imatinib after 12 months of therapy, only one patient did not achieve either CCgR or $B C R-A B L<1 \%$. However, three out of seven evaluable patients did not 
achieve MMR after 12 months of treatment (all patients were analyzed according to the International Scale of MR). Even though this was a small number of patients, the influence of CYP3A activity on the achievement of MMR was analyzed in a prospective setting. However, CYP3A activity (analyzed at the three-month time point) did not have a significant influence on the MMR rates (data not shown).

The prospective set of patients was also used to study a possible induction of CYP3A activity over time. The mean CYP3A metabolic ratio measured before the start of imatinib was 15.4, which was not statistically different from the mean ratio of 14.9 observed three months after treatment start (Fig 4a). Also, the analysis of CGP74588/imatinib ratios after one month (mean: 33 days) and six months (mean: 183 days) of imatinib treatment did not show any significant change (Fig 4b).

Influence of ABCG2 and OCT-1 genotypes on imatinib plasma concentrations

The influence of ABCG2 and OCT-1 genotype on imatinib plasma concentrations was evaluable in 24 patients out of the 34 that had valid trough plasma concentration samplings without the co-administration of quinine. The variant genotypes of ABCG2 421C>A (rs2231142), OCT-1 181C>T (rs12208357), 659G>T (rs36103319), 1222A >G (rs628031), deletion of AAGTTGGT in intron 7 (rs4646281A), and 1260-62 deletion (rs72552763) were found in three or more patients and were investigated in terms of influence on imatinib plasma concentrations (Table 1). Other genotypes were not evaluable due to a lack of patients carrying the variant alleles. The OCT-1 1222A>G and the intronic rs4646281A were significantly associated with imatinib plasma concentration. These two variants also seemed to be in linkage disequilibrium because all patients carrying the variant allele of 1222A>G also carried the variant allele of rs4646281A. Patients homozygous for 1222A and insertion of rs4646281A had a median imatinib plasma concentration of $2244 \mathrm{ng} / \mathrm{mL}$ compared to a median of $976 \mathrm{ng} / \mathrm{mL}$ and $980 \mathrm{ng} / \mathrm{mL}$ in the respective homozygous variant patient groups. None of the other investigated genotypes were significantly associated with imatinib plasma concentration.

\section{Discussion}

The metabolic activity of the CYP3A isoenzyme family is known to be highly variable between individuals, and the potential influence of this variation on imatinib pharmacokinetics and therapeutic outcome in CML has not yet been fully investigated. In this study, we have provided evidence that CYP3A metabolism is not a determinant of imatinib or CGP74588 plasma concentrations or response to therapy in CML patients. 
The quinine metabolic ratio had a 5-fold variation in the investigated population. This observation is concordant with previous studies using quinine or intravenously administered midazolam where the majority of test subjects fall into a range of 4- to 6-fold variation in CYP3A activity. ${ }^{13,27,28}$ However, outliers have been identified and an approximately 12-fold variation in CYP3A activity might be expected. ${ }^{29}$, 30 We cannot rule out a significance of CYP3A activity in individual patients with an extreme metabolic phenotype. In these patients, CYP3A metabolism might become the rate-limiting step of imatinib excretion and have a greater influence on the imatinib plasma concentrations than what was observed in the present study.

No association was found between CYP3A activity and the response to imatinib treatment. Ten patients in our study did not achieve either a $B C R-A B L<1 \%$ or a CCgR on imatinib treatment. In contrast to what was found in our earlier pilot study, these patients did not have a significantly different level of CYP3A activity compared to the responding patients. In line with this finding, CYP3A activity did not influence the occurrence of serious hematologic toxicity during the first year of treatment.

The reason for the discrepancy between the pilot study and the present study might be the limited number of patients in the pilot $(n=14)$ and confirms the importance of validating significant outcomes from small patient cohorts. The increased number of patients in the present follow-up study should more accurately reflect the true relationship between CYP3A activity and imatinib outcome. However, to draw a definitive conclusion with respect to the role of CYP3A activity in imatinib pharmacokinetics, confirmatory results using other probe drugs and patient groups selected based on extreme enzymatic activities might be warranted.

In both the present study and the pilot study, the CYP3A activity for the majority of patients was evaluated retrospectively compared to their first year of treatment that was used as the primary endpoint in both studies. This could potentially influence the outcome of the study if constitutive CYP3A activity changes within individuals over time. The knowledge of intra-individual CYP3A variability over longer periods of time is scarce. However, the intra-individual CYP3A variability over three months is approximately $10 \%,{ }^{31}$ which should be greatly exceeded by the observed inter-individual variability. This is also in agreement with our findings where prospectively included patients did not show any significant changes in CYP3A activity over three months.

The metabolic ratio of quinine/3S-Q was used as a measure of in vivo CYP3A activity because quinine not only shares the metabolic pathway with imatinib but also it shares other important pharmacokinetic properties. Both quinine and imatinib have a high bioavailability, ${ }^{32,33}$ that indicates a relative insensitivity to CYP3A metabolism 
at the intestinal absorption site. This aspect was also illustrated by the fact that quinine pharmacokinetics were not affected by grapefruit juice inhibition of intestinal CYP3A4 activity. ${ }^{34}$ Although CYP3A5 does not account for much of the CYP3A activity in the Swedish population due to the common occurrence of the dysfunctional CYP3A5*3 genotype, ${ }^{21}$ quinine is metabolized by both CYP3A4 and CYP3A5, ${ }^{20,}{ }^{21}$ when expressed, which is also the case for imatinib metabolism. Quinine has previously been validated as a functional probe drug for CYP3A phenotyping, and quinine metabolic ratios correlate with the metabolic ratio of the suggested CYP3A probe omeprazole. $^{19}$

We did not find any influence of imatinib administration on CYP3A activity because patients phenotyped before starting imatinib treatment did not have a significantly different quinine metabolic ratio after three months on imatinib. Conversely, significant interactions were seen the other way around and co-administration of quinine significantly decreased imatinib plasma concentrations and increased the CGP74588/imatinib ratio compared to the second sampling in which quinine was not administered. This finding indicates an induction of CYP3A activity by quinine. This potential interaction between quinine and imatinib has, to our knowledge, not been investigated previously.

The finding that CYP3A enzyme activity does not influence imatinib plasma concentrations or CGP74588/imatinib ratios leads to the speculation that other pharmacokinetic parameters might be more influential on observed plasma drug concentrations. In the initial experiments on imatinib metabolism in human liver microsomes, it was found that other CYP enzymes (CYP1A2, CYP2C8, CYP2C9, CYP2C19 and CYP2D6) are also capable of imatinib metabolism but only to a minor extent. ${ }^{3}$ However, more recent data indicate that the CYP2C8 enzyme might indeed play a role in imatinib metabolism and the formation of CGP74588, potentially contributing to the inter-individual variability in imatinib pharmacokinetics. ${ }^{35}$

Furthermore, the activity of the uptake transport protein OCT-1, measured in blood mononuclear cells, has previously been found to be associated with imatinib outcome. ${ }^{36}$ In addition, OCT-1 is expressed in the sinusoidal membranes of hepatocytes ${ }^{8}$ potentially regulating the accessibility of imatinib for hepatic metabolism. Although the sample size of the present study was not primarily designed for the investigation of genotypes, we decided to test if transporter genotype would be a better predictor of imatinib plasma concentrations than CYP3A activity.

A significant correlation was found between imatinib plasma concentrations and the OCT-1 1222A $>\mathrm{G}$ and deletion in intron 7 (rs4646281A) genotypes. The 1222A allele has previously been associated with efficacy and 
adverse events of metformin in diabetic patients. ${ }^{37,38}$ Similar to our findings, a deletion in OCT-1 intron 7 (rs36056065), linked to the 1222A>G genotype, showed a significant influence on metformin adverse events. ${ }^{38}$ This intronic variant is located only two nucleotides from the rs4646281 variant studied here. Decreased OCT-1 transcript levels of the 1222A allele in the liver were identified, but these differences were not statistically significant. ${ }^{37}$ Decreased OCT-1 activity of the 1222A allele would support our findings of increased imatinib plasma concentration in individuals carrying this allele. These results support previous investigators’ conclusions that OCT-1 activity influences the pharmacokinetics and response to imatinib therapy.

The two polymorphic ATP-binding cassette transporters, ABCB1 and ABCG2, are responsible for hepatic efflux of imatinib. However, the ABCG2 variants most influential on imatinib transport are relatively rare in Caucasian populations and only three individuals heterozygous for ABCG2 421C>A could be investigated in the present study. This ABCG2 variant was not found to associate with imatinib plasma concentration. We have also found previously that CGP74588 is a better substrate for both ABCB1 and ABCG2 than imatinib, ${ }^{39,40}$ which might be one of the reasons for the discordance that we observed between CGP74588/imatinib ratios and CYP3A activity. Due to the limited number of samples in this study we did not investigate ABCB1 genotype.

\section{Conclusion}

This study found that CYP3A enzyme activity, as measured by quinine metabolic ratio, does not correlate with the plasma concentrations of imatinib or CGP74588 and is not predictive of imatinib therapeutic outcome or the hematologic toxicity of imatinib after 12 months of first-line treatment. Also there is no correlation between CYP3A activity and CGP74588/imatinib concentration ratios. These findings suggest that although imatinib is primarily metabolized by CYP3A enzymes, this metabolic activity is not the determinant of the resulting plasma concentrations. The variability in imatinib plasma concentration seems to be due on other pharmacokinetic processes that have yet to be identified.

\section{Acknowledgements}

The authors would like to thank the staff at the hematology departments of the hospitals in Östergötland, Västervik, Jönköping, Örebro, Uppsala, and Lund for their contributions to this study. 


\section{References}

1. de Lavallade H, Apperley JF, Khorashad JS, et al. Imatinib for newly diagnosed patients with chronic myeloid leukemia: incidence of sustained responses in an intention-to-treat analysis. J Clin Oncol. 2008;26:3358-3363.

2. Hochhaus A, Druker B, Sawyers C, et al. Favorable long-term follow-up results over 6 years for response, survival, and safety with imatinib mesylate therapy in chronic-phase chronic myeloid leukemia after failure of interferon-alpha treatment. Blood. 2008;111:1039-1043.

3. Novartis. NDA 21-335, Clinical pharmacology and biopharmaceutics review [US Food and Drug Administration Web site]. April 12, 2001. Available at: http://www.accessdata.fda.gov/drugsatfda_docs/nda/2001/21-335_Gleevec_biopharmr_P1.pdf. Accessed September 1, 2015.

4. Peng B, Lloyd P, Schran H. Clinical pharmacokinetics of imatinib. Clin Pharmacokinet. 2005;44:879-894.

5. Dohse M, Scharenberg C, Shukla S, et al. Comparison of ATP-binding cassette transporter interactions with the tyrosine kinase inhibitors imatinib, nilotinib, and dasatinib. Drug Metab Dispos. 2010;38:1371-1380.

6. Shukla S, Sauna ZE, Ambudkar SV. Evidence for the interaction of imatinib at the transport-substrate site(s) of the multidrug-resistance-linked ABC drug transporters ABCB1 (P-glycoprotein) and ABCG2. Leukemia. 2008;22:445-447.

7. Thomas J, Wang L, Clark RE, et al. Active transport of imatinib into and out of cells: implications for drug resistance. Blood. 2004;104:3739-3745.

8. Koepsell H, Lips K, Volk C. Polyspecific organic cation transporters: structure, function, physiological roles, and biopharmaceutical implications. Pharm Res. 2007;24:1227-1251.

9. Thiebaut F, Tsuruo T, Hamada H, et al. Cellular localization of the multidrug-resistance gene product Pglycoprotein in normal human tissues. Proc Natl Acad Sci U S A. 1987;84:7735-7738.

10. Polgar O, Robey RW, Bates SE. ABCG2: structure, function and role in drug response. Expert Opin Drug Metab Toxicol. 2008;4:1-15.

11. Larson RA, Druker BJ, Guilhot F, et al. Imatinib pharmacokinetics and its correlation with response and safety in chronic-phase chronic myeloid leukemia: a subanalysis of the IRIS study. Blood. 2008;111:4022-4028. 12. Picard S, Titier K, Etienne G, et al. Trough imatinib plasma levels are associated with both cytogenetic and molecular responses to standard-dose imatinib in chronic myeloid leukemia. Blood. 2007;109:3496-3499. 
13. Lamba JK, Lin YS, Schuetz EG, et al. Genetic contribution to variable human CYP3A-mediated metabolism. Adv Drug Deliv Rev. 2002;54:1271-1294.

14. Wilkinson GR. Cytochrome P4503A (CYP3A) metabolism: prediction of in vivo activity in humans. $J$ Pharmacokinet Biopharm. 1996;24:475-490.

15. Green H, Skoglund K, Rommel F, et al. CYP3A activity influences imatinib response in patients with chronic myeloid leukemia: a pilot study on in vivo CYP3A activity. Eur J Clin Pharmacol. 2010;66:383-386. 16. Muller MC, Cross NC, Erben P, et al. Harmonization of molecular monitoring of CML therapy in Europe. Leukemia. 2009;23:1957-1963.

17. Hanfstein B, Muller MC, Hehlmann R, et al. Early molecular and cytogenetic response is predictive for long-term progression-free and overall survival in chronic myeloid leukemia (CML). Leukemia. 2012;26:20962102.

18. Hehlmann R, Lauseker M, Jung-Munkwitz S, et al. Tolerability-adapted imatinib 800 mg/d versus 400 mg/d versus $400 \mathrm{mg} / \mathrm{d}$ plus interferon-alpha in newly diagnosed chronic myeloid leukemia. J Clin Oncol. 2011;29:1634-1642.

19. Mirghani RA, Ericsson O, Tybring G, et al. Quinine 3-hydroxylation as a biomarker reaction for the activity of CYP3A4 in man. Eur J Clin Pharmacol. 2003;59:23-28.

20. Allqvist A, Miura J, Bertilsson L, et al. Inhibition of CYP3A4 and CYP3A5 catalyzed metabolism of alprazolam and quinine by ketoconazole as racemate and four different enantiomers. Eur J Clin Pharmacol. 2007;63:173-179.

21. Mirghani RA, Sayi J, Aklillu E, et al. CYP3A5 genotype has significant effect on quinine 3-hydroxylation in Tanzanians, who have lower total CYP3A activity than a Swedish population. Pharmacogenet Genomics. 2006;16:637-645.

22. Stevens JC, Hines RN, Gu C, et al. Developmental expression of the major human hepatic CYP3A enzymes. J Pharmacol Exp Ther. 2003;307:573-582.

23. Mirghani RA, Ericsson O, Cook J, et al. Simultaneous determination of quinine and four metabolites in plasma and urine by high-performance liquid chromatography. J Chromatogr B Biomed Sci Appl. 2001;754:5764.

24. Parise RA, Ramanathan RK, Hayes MJ, et al. Liquid chromatographic-mass spectrometric assay for quantitation of imatinib and its main metabolite (CGP 74588) in plasma. J Chromatogr B Analyt Technol Biomed Life Sci. 2003;791:39-44. 
25. Flockhart. Drug Interactions: Cytochrome P450 Drug Interaction Table [Indiana University School of Medicine Web site]. 2007. Available at: http://medicine.iupui.edu/clinpharm/ddis/main-table/. Accessed September 1, 2015.

26. Liu YT, Hao HP, Liu CX, et al. Drugs as CYP3A probes, inducers, and inhibitors. Drug Metab Rev. 2007;39:699-721.

27. Ingelman-Sundberg M, Sim SC, Gomez A, et al. Influence of cytochrome P450 polymorphisms on drug therapies: pharmacogenetic, pharmacoepigenetic and clinical aspects. Pharmacol Ther. 2007;116:496-526. 28. Floyd MD, Gervasini G, Masica AL, et al. Genotype-phenotype associations for common CYP3A4 and CYP3A5 variants in the basal and induced metabolism of midazolam in European- and African-American men and women. Pharmacogenetics. 2003;13:595-606.

29. Rodriguez-Antona C, Sayi JG, Gustafsson LL, et al. Phenotype-genotype variability in the human CYP3A locus as assessed by the probe drug quinine and analyses of variant CYP3A4 alleles. Biochem Biophys Res Commun. 2005;338:299-305.

30. Lin YS, Lockwood GF, Graham MA, et al. In-vivo phenotyping for CYP3A by a single-point determination of midazolam plasma concentration. Pharmacogenetics. 2001;11:781-791.

31. Kashuba AD, Bertino JS, Jr., Rocci ML, Jr., et al. Quantification of 3-month intraindividual variability and the influence of sex and menstrual cycle phase on CYP3A activity as measured by phenotyping with intravenous midazolam. Clin Pharmacol Ther. 1998;64:269-277.

32. Peng B, Dutreix C, Mehring G, et al. Absolute bioavailability of imatinib (Glivec) orally versus intravenous infusion. J Clin Pharmacol. 2004;44:158-162.

33. Paintaud G, Alvan G, Ericsson O. The reproducibility of quinine bioavailability. Br J Clin Pharmacol. 1993;35:305-307.

34. Ho PC, Chalcroft SC, Coville PF, et al. Grapefruit juice has no effect on quinine pharmacokinetics. Eur J Clin Pharmacol. 1999;55:393-398.

35. Nebot N, Crettol S, d'Esposito F, et al. Participation of CYP2C8 and CYP3A4 in the N-demethylation of imatinib in human hepatic microsomes. Br J Pharmacol. 161:1059-1069.

36. White DL, Radich J, Soverini S, et al. Chronic phase chronic myeloid leukemia patients with low OCT-1 activity randomized to high-dose imatinib achieve better responses and have lower failure rates than those randomized to standard-dose imatinib. Haematologica. 2012;97:907-914. 
37. Shikata E, Yamamoto R, Takane H, et al. Human organic cation transporter (OCT1 and OCT2) gene polymorphisms and therapeutic effects of metformin. J Hum Genet. 2007;52:117-122.

38. Tarasova L, Kalnina I, Geldnere K, et al. Association of genetic variation in the organic cation transporters OCT1, OCT2 and multidrug and toxin extrusion 1 transporter protein genes with the gastrointestinal side effects and lower BMI in metformin-treated type 2 diabetes patients. Pharmacogenet Genomics. 2012;22:659-666. 39. Skoglund K, Boiso Moreno S, Jonsson JI, et al. Single-nucleotide polymorphisms of ABCG2 increase the efficacy of tyrosine kinase inhibitors in the K562 chronic myeloid leukemia cell line. Pharmacogenet Genomics. 2014;24:52-61.

40. Skoglund K, Moreno SB, Baytar M, et al. ABCB1 haplotypes do not influence transport or efficacy of tyrosine kinase inhibitors in vitro. Pharmgenomics Pers Med. 2013;6:63-72. 


\section{Figure legends}

Fig 1 The influence of CYP3A metabolic activity on patient response to imatinib 12 months after start of treatment. In a), patients that achieved an optimal response (CCgR or $B C R-A B L<1 \%$ ) were compared to patients that did not achieve this level of response $(n=43)$. In $\mathbf{b})$ and $\mathbf{c})$, the CYP3A activity was compared between patients that had CCgR $(n=38)$ and MMR $(n=37)$ versus the respective non-responding patients. Horizontal lines represent median values. $\mathrm{CCgR}=$ complete cytogenetic response, $\mathrm{MMR}=$ major molecular response.

Fig 2 Influence of CYP3A activity on hematologic toxicity during the first 12 months of $400 \mathrm{mg} /$ day imatinib in patients who did and did not experience grade 3-4 hematological toxicity $(n=36)$. Horizontal lines represent the median CYP3A activity.

Fig 3 Correlation of CYP3A metabolic activity to a) imatinib plasma concentration and b) the CGP74588/imatinib ratio in plasma $(n=34)$. Solid lines represent the linear regression and dotted lines represent the $95 \%$ confidence intervals.

Fig 4 Induction of CYP3A activity over time during the course of imatinib treatment. a) CYP3A activity measured before and three months after the start of imatinib treatment $(n=7)$. b) The ratio of CGP74588 and imatinib plasma concentrations measured after one and six months of imatinib treatment $(\mathrm{n}=7)$. 
Table 1: Influence of OCT-1 and ABCG2 genotype on imatinib plasma concentration

\begin{tabular}{|c|c|c|c|c|c|c|c|c|c|c|}
\hline \multirow[b]{2}{*}{$\begin{array}{l}\text { Variant } \\
\text { rs\# }\end{array}$} & \multicolumn{3}{|c|}{ Referent genotype } & \multicolumn{3}{|c|}{ Heterozygous } & \multicolumn{3}{|c|}{ Variant genotype } & \multirow[b]{2}{*}{$\begin{array}{c}P \text { - } \\
\text { value }^{\mathrm{a}}\end{array}$} \\
\hline & Genotype & $\mathbf{N}$ & $\begin{array}{l}\text { Imatinib } \\
\text { [median } \\
\text { (range)] } \\
\text { (ng/mL) }\end{array}$ & Genotype & $\mathbf{N}$ & $\begin{array}{l}\text { Imatinib } \\
\text { [median } \\
\text { (range)] } \\
\text { (ng/mL) }\end{array}$ & Genotype & $\mathbf{N}$ & $\begin{array}{l}\text { Imatinib } \\
\text { [median } \\
\text { (range)] } \\
\text { (ng/mL) }\end{array}$ & \\
\hline \multicolumn{11}{|l|}{ OCT-1 } \\
\hline rs12208357 & CC & 21 & $\begin{array}{c}983 \\
(509-3485)\end{array}$ & CT & 3 & $\begin{array}{c}1193 \\
(1069-1955)\end{array}$ & $\mathrm{TT}$ & 0 & n.a. & 0.401 \\
\hline rs36103319 & GG & 5 & $\begin{array}{c}889 \\
(509-1955)\end{array}$ & GT & 15 & $\begin{array}{c}1193 \\
(524-3485)\end{array}$ & TT & 0 & n.a. & 0.230 \\
\hline rs4646281A & II & 3 & $\begin{array}{c}2244 \\
(1400-3485)\end{array}$ & ID & 11 & $\begin{array}{c}1069 \\
(509-3207)\end{array}$ & DD & 10 & $\begin{array}{c}980 \\
(524-1387)\end{array}$ & $0.047^{\mathrm{b}}$ \\
\hline rs628031 & AA & 3 & $\begin{array}{c}2244 \\
(1400-3485)\end{array}$ & AG & 11 & $\begin{array}{c}1069 \\
(509-3207)\end{array}$ & GG & 9 & $\begin{array}{c}976 \\
(524-1387)\end{array}$ & $0.047^{\mathrm{b}}$ \\
\hline rs72552763 & II & 12 & $\begin{array}{c}993 \\
(509-3485)\end{array}$ & ID & 10 & $\begin{array}{c}1026 \\
(720-3207)\end{array}$ & $\mathrm{DD}$ & 1 & $\begin{array}{l}1144 \\
\text { (n.a.) }\end{array}$ & $0.525^{c}$ \\
\hline \multicolumn{11}{|l|}{ ABCG2 } \\
\hline rs2231142 & CC & 21 & $\begin{array}{c}1096 \\
(509-3207)\end{array}$ & CA & 3 & $\begin{array}{c}951 \\
(795-3485)\end{array}$ & AA & 0 & n.a. & 1.000 \\
\hline $\begin{array}{l}\text { N: number } \\
\text { a'Mann-Whi } \\
\text { b'Kruskal-W } \\
\text { 'Statistics w } \\
\text { patients gro }\end{array}$ & $\begin{array}{l}\text { patients; I: } \\
\text { ley 2-sampl } \\
\text { llis test } \\
\text { re analyzed } \\
\text { ped togethe }\end{array}$ & on & int D: deletio & $\begin{array}{l}\text { n.a.: not av } \\
\text { us for the re }\end{array}$ & 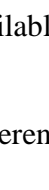 & enotype vers & heterozygo & $=$ & omozygous & ant \\
\hline
\end{tabular}




\section{Supplemental Digital Content}

Supplemental digital content 1.pdf

Supplemental digital content 2.pdf 
Fig 1
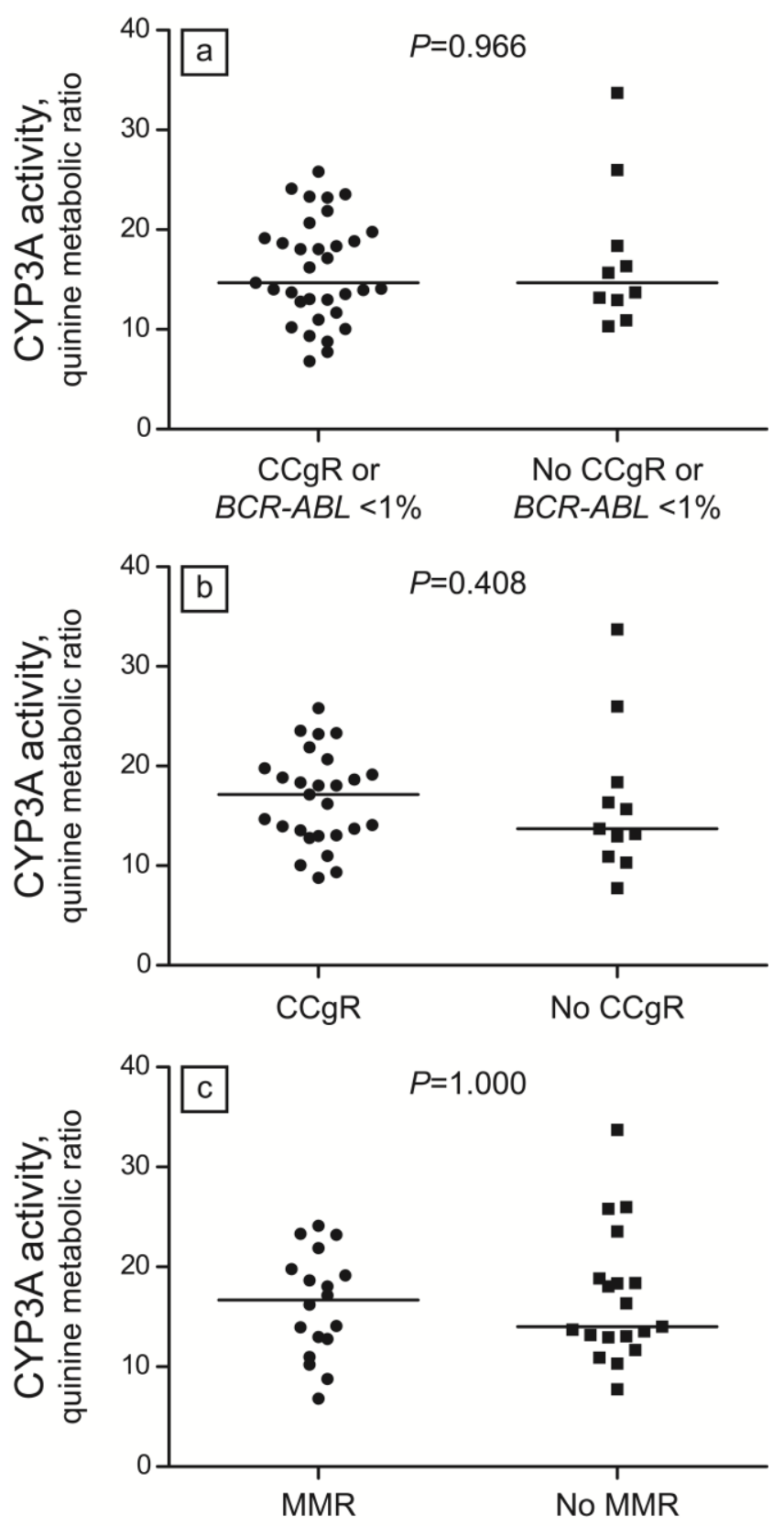
Fig 2

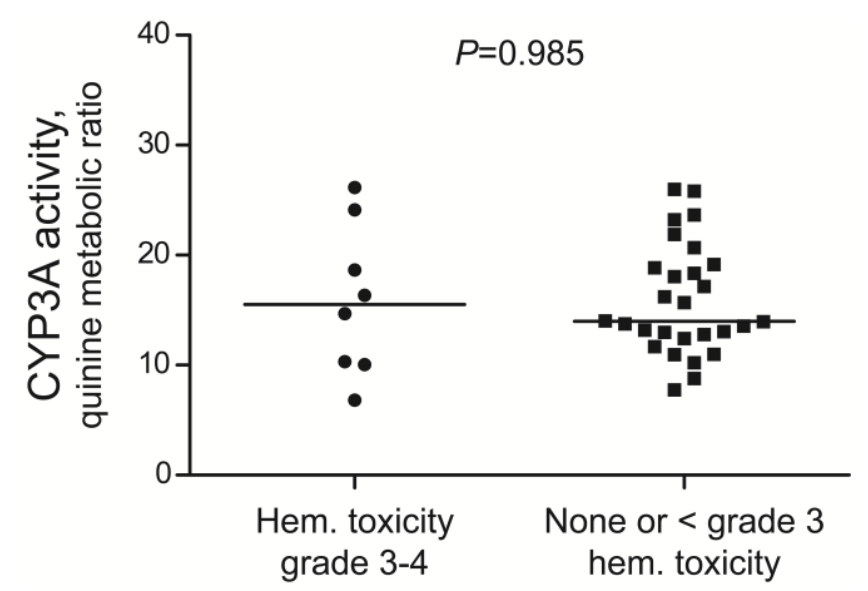

Fig 3
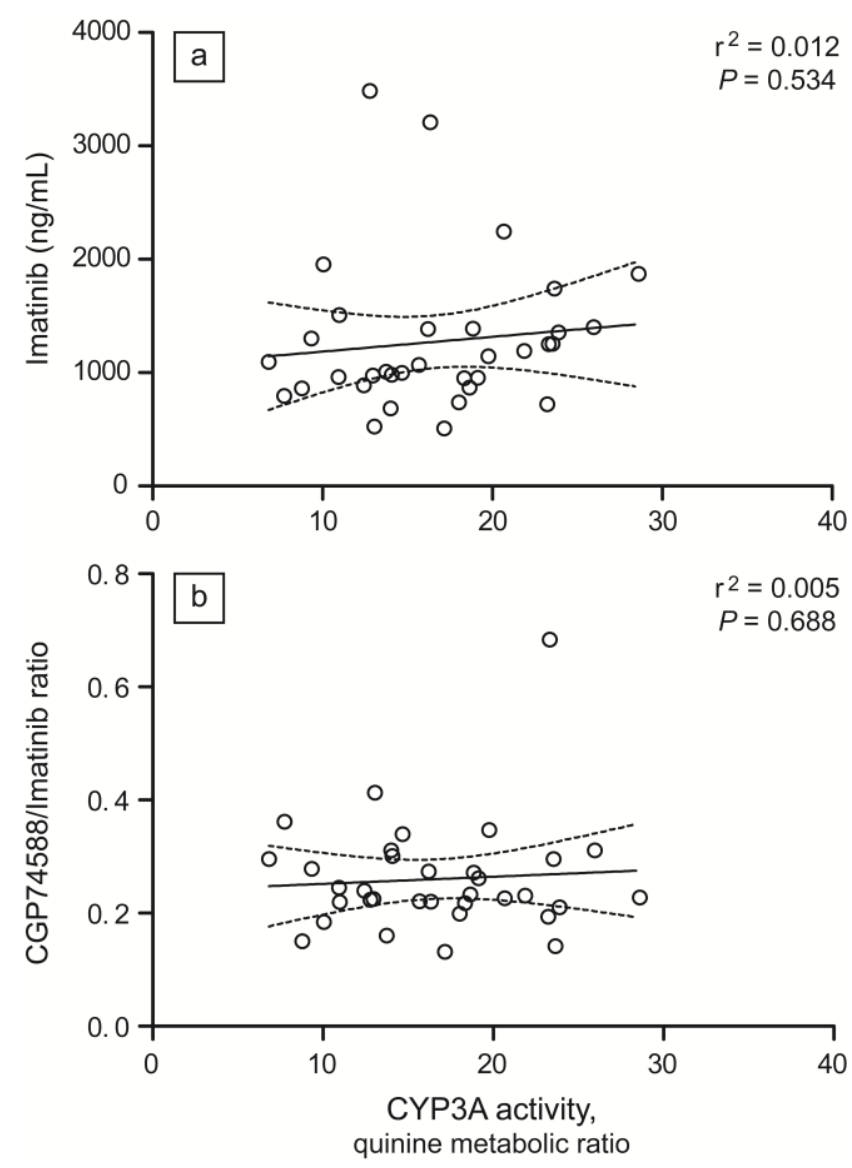
Fig 4
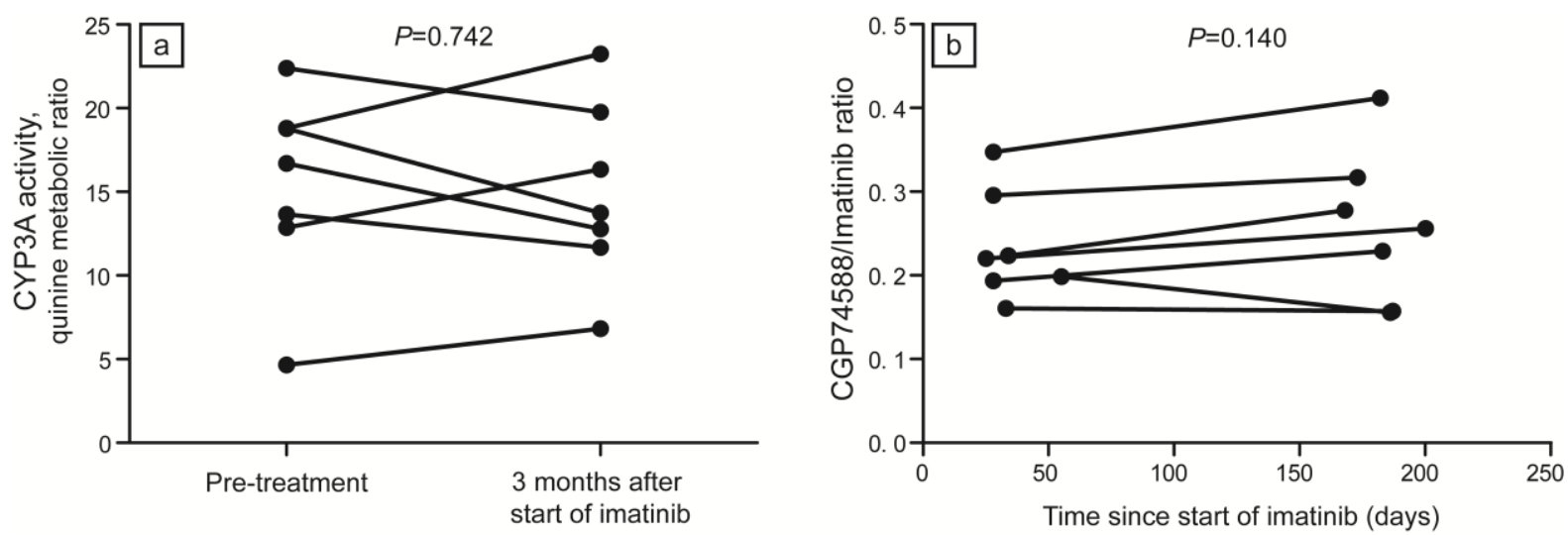